\title{
Consistent Combination of Satellite and Terrestrial Gravity Field Observations in Regional Geoid Modeling: A Case Study for Austria
}

\author{
Christian Pock, Torsten Mayer-Guerr, and Norbert Kuehtreiber
}

\begin{abstract}
In this investigation a consistent combination of the complementary data types of satellite observations and the available terrestrial gravity field measurements in Austria is considered. For this purpose, the well known Remove-Compute-Restore technique is adapted to perform long- and short-wavelength signal reductions. The long-wavelength effect is represented by a global satellite-only model in terms of spherical harmonics. The shortwavelength are modeled by topographic masses in the spatial domain. As the topographic reduction contains also long-wavelength effects a possible double consideration has to be avoided. Alternatively to Least Squares Collocation (LSC) method (Moritz 1980a) a least squares approach with parametrization as Radial Basis Functions (RBF) is applied. The $\mathrm{RBF}$ approach has the advantage that an increasing number of observations can be included in the calculations and a downsampling of the available data, as it is required in LSC, will no longer be necessary. Another advantage is that RBF is to able to handle an inhomogeneous input data distribution. The very first outcomes are verified by comparing with independent GPS/leveling observations.
\end{abstract}

\section{Keywords}

GPS/leveling observations - Radial basis functions (RBF) - Regional geoid modeling

- Remove-compute-restore technique

\section{Introduction}

The determination of the Earth's gravity field can take place on different spatial scales. Dedicated satellite missions like CHAMP (CHAllenging Minisatellite Payload) (Reigber et al. 2002), GRACE (Gravity Recovery And Climate Experiment) (Tapley et al. 2002) or GOCE (Gravity field and steady-state Ocean Circulation Explorer) (Drinkwater et al. 2003) detect the global gravity field with high accuracy. For

C. Pock $(\bowtie) \cdot$ T. Mayer-Guerr

Institute of Theoretical Geodesy and Satellite Geodesy, Steyrergasse 30/III, 8010 Graz, Austria

e-mail: christian.pock@tugraz.at; mayer-guerr@tugraz.at

N. Kuehtreiber

Institute of Navigation, Steyrergasse 30/III, 8010 Graz, Austria

e-mail: norbert.kuehtreiber@tugraz.at instance, with the GOCE mission it is possible to derive a global gravity field model parametrized in terms of a spherical harmonic series expansion up to a degree and order (D/O) of 250 corresponding to a spatial resolution of approximately $80 \mathrm{~km}$ half wavelength. The accuracy in terms of geoid height with $100 \mathrm{~km}$ spatial resolution is $1-2 \mathrm{~cm}$ (Drinkwater et al. 2008). However, for regional applications the spatial resolution of a satellite-only gravity field model is insufficient. Many local and regional applications require a much higher spatial resolution than a satellite-only model can provide.

On the other hand, local gravity field models derived from terrestrial and airborne gravity field data e.g. gravity anomalies or deflections of the vertical reflect the small scale features better as the satellite data but lack from long-wavelength information. Therefore a pure gravimetric geoid solution is affected by long-wavelength errors (Pail et al. 2009). 
So an consistent combination of satellite derived global gravity field models with complementary local gravity field data suggests itself. Recently two research projects GEOnAUT (Pail et al. 2008) and GEOID + (Kuehtreiber et al. 2011) were carried out at Graz University of Technology. By combining heterogeneous data the precision of the Austrian geoid has improved to sub-decimeter level. The computation was based on the Remove-Compute-Restore technique. In the remove step a global satellite only model has been used to represent the long-wavelength components as well as a topographic reduction has been carried out to remove the topographic short-wavelength components from the measured gravity. For geoid computation the LSC approach was used and furthermore all removed components have been restored afterwards. Nevertheless, the present Austrian geoid solution (Pail et al. 2008) shows long wavelength errors when compared to the GPS/leveling observations which is supposed to be a problem of the combination of the satellite derived gravity field models and the terrestrial gravity data. A common practical approach to handle this problem is to introduce a non-physical correction surface. The need of such a correction surface for practical applications was tried to be avoided by combinations with global satelliteonly models of different $\mathrm{D} / \mathrm{O}$. However, these attempts were not successful and a correction surface is still required. The main goal of this investigation is to discover a proper way for the combination of the satellite derived global gravity field models with the complementary terrestrial data with the result, that the computed geoid can be directly validated with GPS/leveling observations and a correction surface is not needed anymore.

\section{Data}

\subsection{Gravity Dataset}

The achieved results are based on a gravity dataset spanning Central Europe. This database was established by Norbert Kuehtreiber during the last decades and the gravity measurements have been provided by several project partners (e.g. Federal Office of Meteorology and Surveying (BEV), Institute of Geophysics (Mining University Leoben) or the Austrian Mineral Oil Administration (OMV). Including Austrian data and data from neighbouring countries there are currently almost 123,000 measurements available. For these first investigations only 28,212 gravity measurements within Austria have been used to avoid datum problems. In the future all available data will be incorporated. The spatial distribution of the data is about 1 to $15 \mathrm{~km}$ and quite inhomogeneous. In mountainous regions measurements are spare whereas in valleys and flatland a huge amount of measurements is available. The gravity data consists of

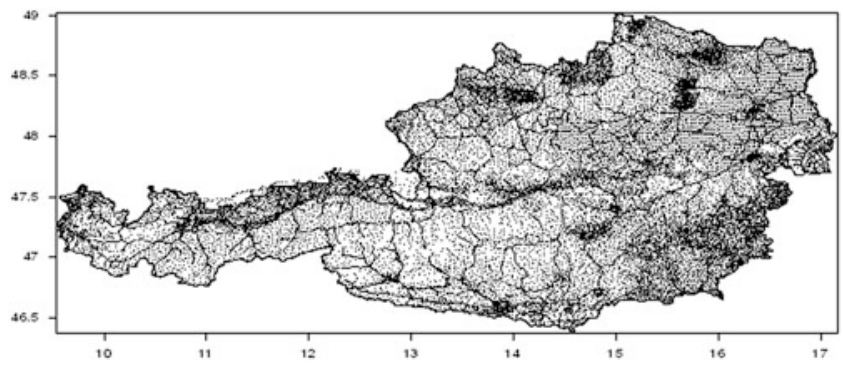

Fig. 1 Sample of 28212 gravity measurements

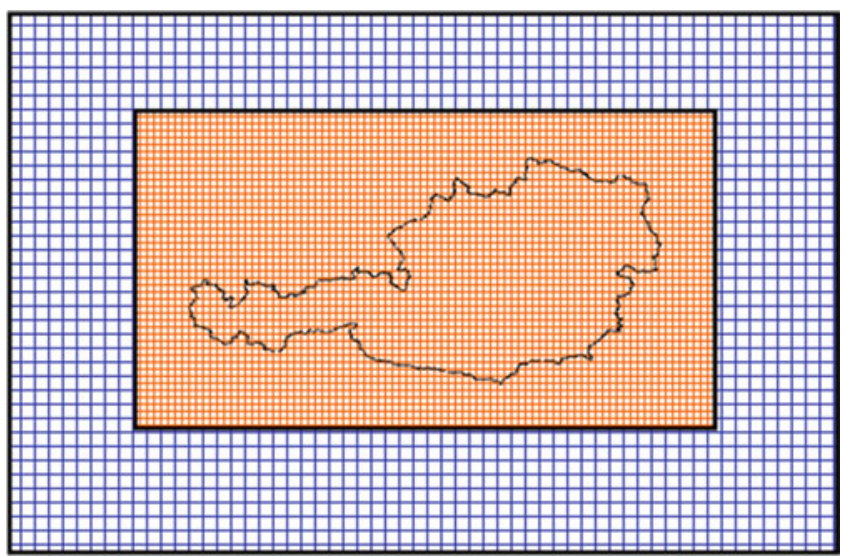

Fig. 2 Combination of the two DTMs in the Central European regioncoarse (blue), dense (red)

global WGS-84 coordinates and the heights are related to the official Austrian orthometric height system. In Fig. 1 the data sample is shown.

\subsection{Digital Terrain Model (DTM)}

The topographic reduction which is representing the shortwavelengths components of the gravity field is based on two DTMs established by the BEV. The resolution for the coarse DTM is $11.25^{\prime \prime} \times 18.75^{\prime \prime}$. For the dense DTM a uniform resolution of $1.40625^{\prime \prime} \times 2.34375^{\prime \prime}$ is provided. Both DTMs cover the Central European area. Further information concerning the development of the height models can be found in Graf (1996) or Ruess (1983). The DTMs have to be combined in the calculation. Therefore the coarse DTM covers the whole area. In the area nearby Austria the coarse grid is refined by a residual dense grid which contains not the complete topography but only the corrections to the coarse grid (Fig. 2).

\subsection{GPS/Leveling Observations}

The GPS/leveling observations have been used to check the quality of the computed geoid solution. Currently 192 points 
with measured geoid heights are available within Austria. This set is composed of points from the Austrian Reference Frame-net (AREF-net) and GPS permanent stations provided by the BEV. The precision of the observed geoid heights is assumed to be a few centimeter.

\section{Consistent Reduction}

\subsection{Remove Step}

The remove procedure is used to smooth the measured gravity field quantities to permit the continuation and interpolation to the generating geoid. Therefore the measured data on Earth surface has to be reduced by long- and short wavelength components. The long wavelength part is known by means of a given global satellite model which is expressed in terms of spherical harmonic expansion. The short wavelength part is a function of the mass and density distribution of the topography and can be approximated by the DTM. The effect of the topography is represented by prism integrated in the spatial domain and contains in principle all frequencies and also some long-wavelength part which is already considered in the satellite data. Therefore the effect of the DTM have to be reduced by this long-wavelength part and is expressed as a spherical harmonic expansion limited by the maximum degree of the satellite derived global gravity field model. The topographic coefficients are obtained by:

$$
\left(\begin{array}{l}
c_{n m} \\
s_{n m}
\end{array}\right)=\frac{1}{M(2 n+1)} \iiint_{\Omega}\left(\frac{r^{\prime}}{R}\right)^{n}\left(\begin{array}{l}
C_{n m}\left(\theta^{\prime}, \lambda^{\prime}\right) \\
S_{n m}\left(\theta^{\prime}, \lambda^{\prime}\right)
\end{array}\right) \rho\left(r^{\prime}\right) d \Omega
$$

where in Eq. (1) the $C_{n m}$ and $S_{n m}$ are representing the spherical harmonics as defined

$$
\left(\begin{array}{c}
C_{n m} \\
S_{n m}
\end{array}\right)=P_{n m}(\cos \vartheta)\left(\begin{array}{c}
\cos m \lambda \\
\sin m \lambda
\end{array}\right)
$$

The Earth mass is denoted with $M$. The $\rho$ stands for the standard crustal density. For this investigation a crustal density of $\rho=2,670 \mathrm{~kg} / \mathrm{m}^{3}$ was chosen. More details about the modeling of the topographic effects in spectral domain can be found in (Rummel et al. 1988). To sum up, the topographic reduction by prism is reduced of long wavelength parts represented by spherical harmonics [Eq. (3)]. The same reduction has to be done for both used DTMs (see Sect. 2.2).

\subsection{Realization of Reduction}

The input gravity dataset consists of 28,212 gravity measurements as described in Sect. 2.1. The long-wavelength part is represented by the latest Gravity Observation Combination

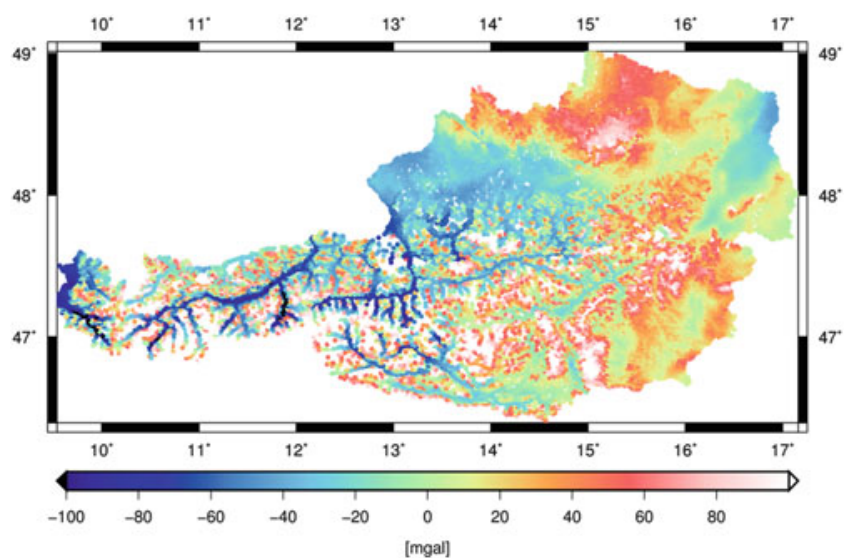

Fig. 3 Free air anomalies $g_{a b s}-\gamma ; \mathrm{rms}=41.56 \mathrm{mgal}$

(GOCO) model GOCO03s (Mayer-Guerr et al. 2012) up to the full degree of 250. For short wavelength reduction the well known prism formula (Wild-Pfeiffer 2007) has been used. The reduction is done without any isostatic compensation. We expect the isostatic part as information which is already included in the global satellite models characterized by the long-wavelength. The reduced gravity $\Delta g_{\text {red }}$ is computed by

$$
\begin{array}{r}
\Delta g_{\text {red }}=g_{\text {abs }}-\gamma-\left[\Delta g_{\text {global }}-\left(g_{\text {Coarse }}-g_{\text {CoarseSHM }}\right)\right. \\
\left.-\left(g_{\text {Dense }}-g_{\text {DenseSHM }}\right)\right] .
\end{array}
$$

The parameter $g_{a b s}$ represents the measured absolute gravity on Earth surface. In a first step the normal gravity $\gamma$ which belongs to the GRS80 (Moritz 1980b) is subtracted from measured gravity data. For the absolute gravity the orthometric height above the geoid is known and the normal gravity is computed at height above the ellipsoid. Therefore the differences are the classical free air anomalies $\Delta g_{f a}$ shown in Fig. 3.

In a next step the long-wavelength reduction $\Delta g_{\text {global }}$ using the GOCO03s global gravity field model has been carried out. The spherical harmonic expansion was performed up to the full degree $n=250$. The rms is now increasing to $47.26 \mathrm{mgal}$ and the remaining topographic parts are clearly show up and can be seen in Fig. 4. This fact is attributable to the spatial distribution of the measured gravity observations. They are mostly measured in valleys and not in mountainous regions and the global model can not resolve the sharp structure of the valleys.

The remaining notations in Eq. (3) are all related to the DTM. The $g_{\text {Coarse }}, g_{\text {Dense }}$ parts are representing the topographic reduction and the $g_{\text {CoarseSHM }}, g_{\text {DenseSHM }}$ terms are the corrections obtained from the spherical harmonic expansion of the DTM. In Fig. 5 the impact of the coarse grid is taken into account. In this illustration and in Table 1 the 


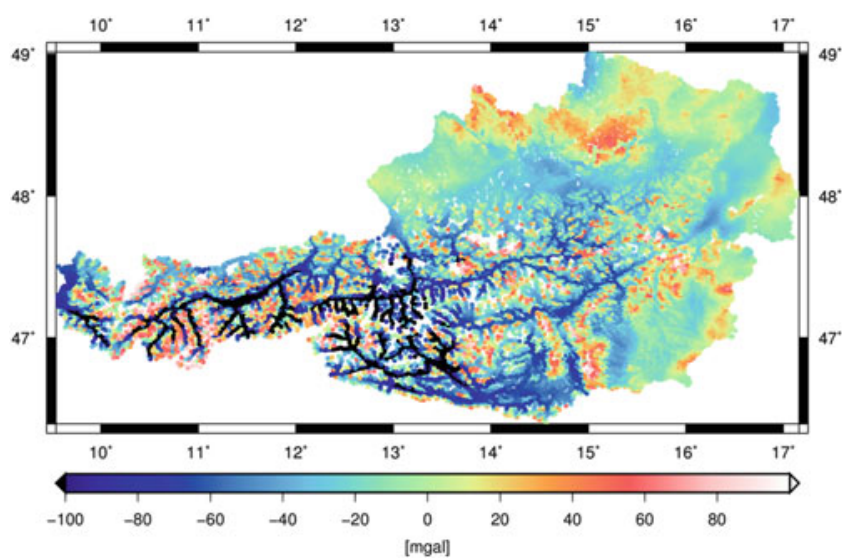

Fig. 4 Additionally reduced by the global part using the GOCO03s model; $g_{a b s}-\gamma-\Delta g_{\text {global }} ; \mathrm{rms}=47.26 \mathrm{mgal}$

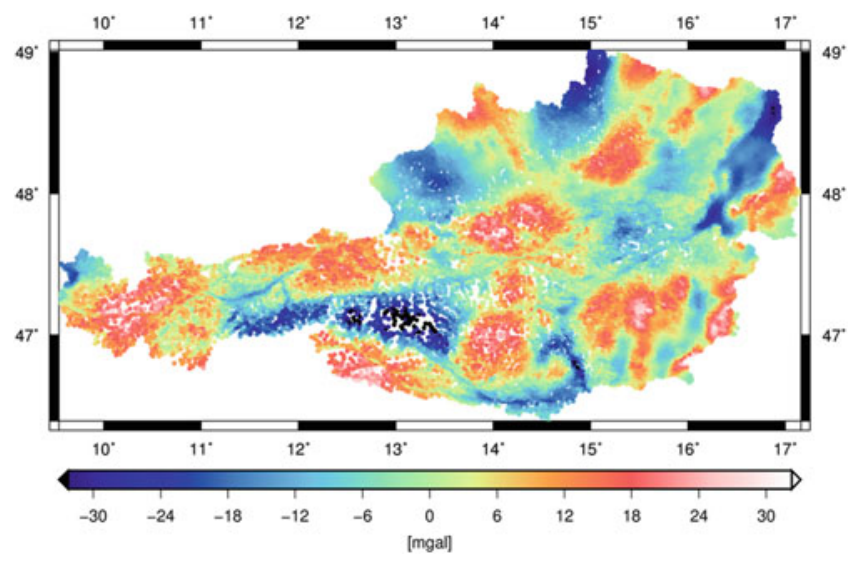

Fig. 5 Additionally reduced by the coarse topographic parts; $g_{a b s}-\gamma$ $\Delta g_{\text {global }}-\left(g_{\text {Coarse }}-g_{\text {CoarseSHM }}\right) ; \mathrm{rms}=11.25 \mathrm{mgal}$

Table 1 Statistics of reduction-adding another reduction step subsequently

\begin{tabular}{lcccl}
\hline & Min [mgal] & Max [mgal] & Mean [mgal] & rms [mgal] \\
\hline$\Delta g_{\text {fa }}$ & -123.78 & 205.42 & 5.84 & 41.56 \\
\hline$\Delta g_{\text {global }}$ & -186.82 & 134.16 & -23.57 & 47.26 \\
\hline$g_{\text {coarse }}$ & -44.64 & 41.39 & -1.32 & 11.25 \\
\hline$g_{\text {dense }}$ & -48.71 & 38.11 & -1.21 & 11.06 \\
\hline
\end{tabular}

effect of the topographic reduction $g_{\text {Coarse }}$ is shown together with the associated coarse DTM derived gravity correction $g_{\text {CoarseSHM }}$ which is computed with help of Eq. (1). The rms is now decreasing to $11.25 \mathrm{mgal}$. In Fig. 6 the impact of the reduction steps using the dense DTM can be seen. In fact, it is rather low compared to Fig. 5 because the dense DTM can be seen as an improvement to the coarse DTM in the overlapping area (see Sect. 2.2). The rms is slightly decreasing to $11.06 \mathrm{mgal}$.

Finally the reduced gravity [see Eq. (3)] is obtained using a proper combination of global and local data and is representing the remaining part from measured absolute

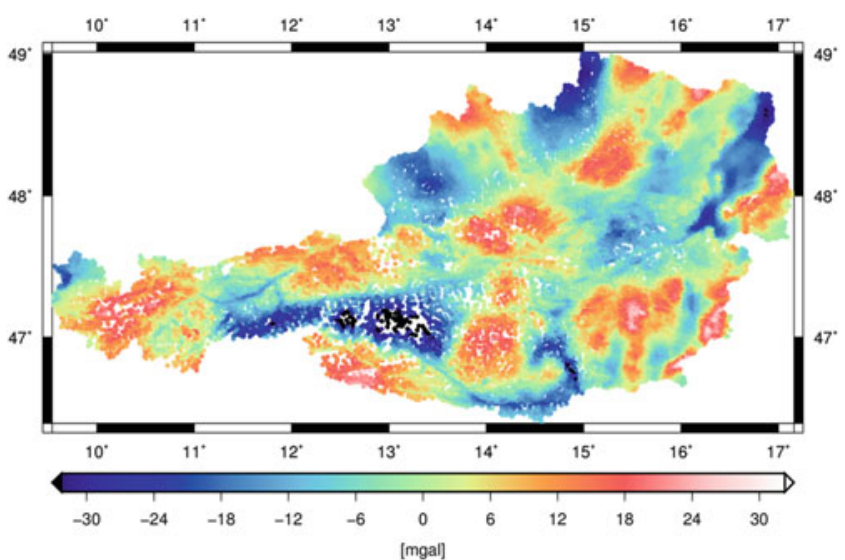

Fig. 6 Reduced gravity $\Delta g_{\text {red }}$ after the final reduction step; $r m s=$ $11.06 \mathrm{mgal}$

gravity on Earth surface within Austria. To give an overview about the impact of every single reduction step the statistical results are summarized in Table 1.

\section{Computation Step: Radial Basis Functions (RBF)}

For the computation step a least squares approach (GaussMarkov model) in association with radial basis functions is introduced. This procedure enables the possibility to estimate the residual parts as supplements to the satellite model. Applying this approach for regional geoid modeling which is based on (Eicker 2008) the gravity anomalies can be represented as series expansion of radial basis functions

$$
\Delta g_{\text {red }}=\sum_{i=1}^{N} a_{i} \phi\left(x, x_{i}\right)
$$

where $a_{i}$ are the unknown scaling coefficients to determine, $x$ denotes the evaluation point and the $x_{i}$ stands for the centers of the basis function. The radial symmetric basis functions $\phi$ which are representing the deviations of the achieved solution compared to a GOCO based solution in turn can be expressed by a sum of Legendre polynomials

$$
\phi\left(x, x_{i}\right)=\frac{G M}{R} \sum_{n=2}^{\infty} \sum_{m=-n}^{n}\left(\frac{n-1}{R}\right) k_{n} Y_{n m}(x) Y_{n m}\left(x_{i}\right) .
$$

The spherical harmonics are denoted as $Y_{n m}$. The shape of the function is controlled by the coefficients $k_{n}$

$$
k_{n}=\frac{\sigma_{n}}{\sqrt{2 n+1}} .
$$




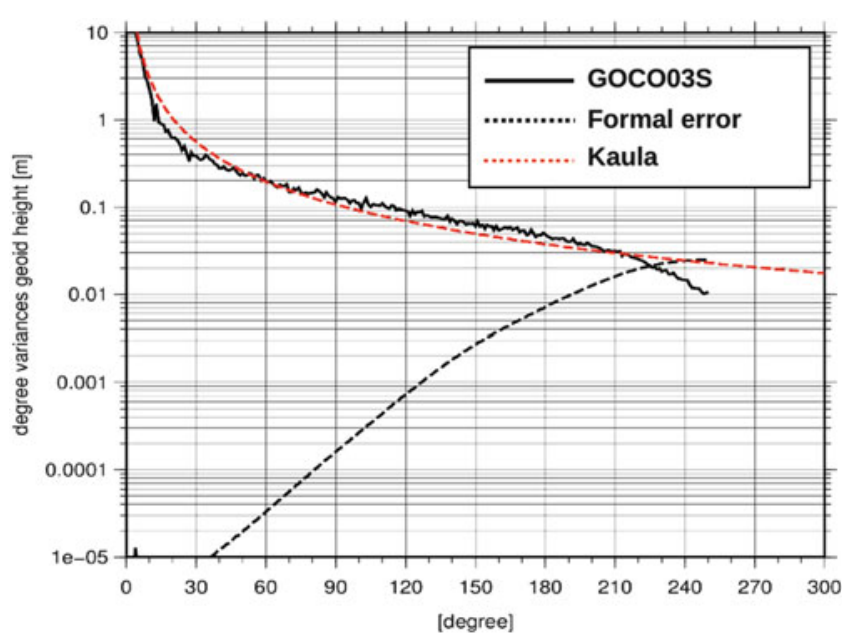

Fig. 7 Transition between the GOCO formal errors and the full signal of the high frequencies using Kaula's rule

For the coefficient up to degree $n=250$ the formal errors of the GOCO03s model have been used. Beyond this degree the coefficients are padded by Kaula's rule:

$$
k_{n}=\frac{1}{n^{2}}
$$

This choice of the shape functions consider the fact, that the satellite model does not affords the full spectral power in the high degrees but provide a high accuracy in the long wavelength and increasingly weaker accuracy with increasing degree. Beyond D/O 250 the high frequencies are still containing the full signal expressed by Kaula's rule. This approach is shown in Fig. 7.

For the spatial distribution of the RBF a global triangle vertex (Schmidt 1981) has been used. The point distribution of the RBF is essential for the outcomes and was chosen homogeneously. In case of regional geoid modeling RBF are situated within Austria and $10 \mathrm{~km}$ beyond Austrian borders to reduce edge effects. To achieve a proper RBF representation a similar spatial resolution as the input data should be ensured. This means that the $k_{n}$ for this investigation are given up to $n=6,000$. This degree corresponds to a point distance of approximately $3 \mathrm{~km}$ on Earth surface and results in 7,091 unknowns.

\section{Results: Restore Step}

In the restore step the operations of the remove step described in Sect. 3.2 have to be reversed. An advantage of the used least squares approach is the overdetermination, which allows the analysis of the residuals and furthermore an a-posteriori variance can be estimated. In Fig. 8 the computed residuals between the reduced input gravity and

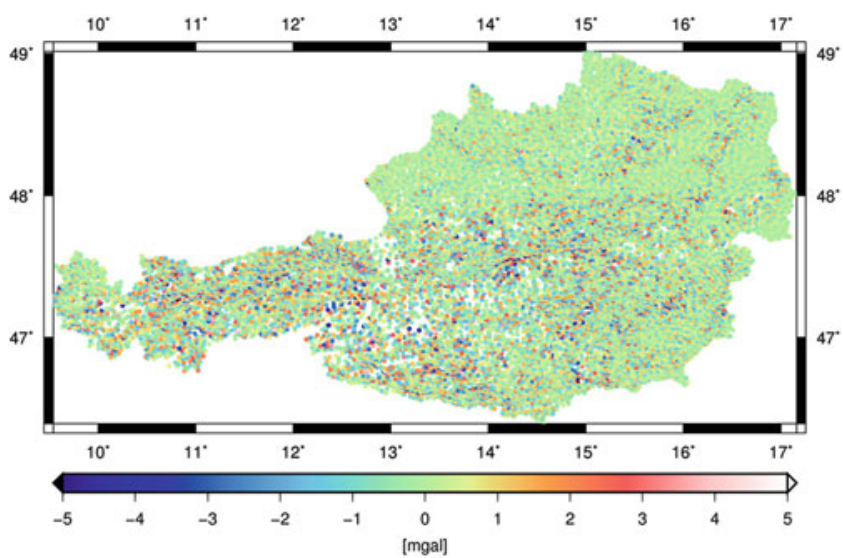

Fig. 8 Residuals between reduced input gravity and estimated gravity based on RBF parametrization; rms $=1.12 \mathrm{mgal}$

Table 2 Statistics of 28,212 residuals

\begin{tabular}{lllll}
\hline & Min [mgal] & Max [mgal] & Mean [mgal] & rms [mgal] \\
\hline$v_{\Delta g_{\text {red }}}$ & -5.77 & 12.14 & -0.01 & 1.12 \\
\hline
\end{tabular}

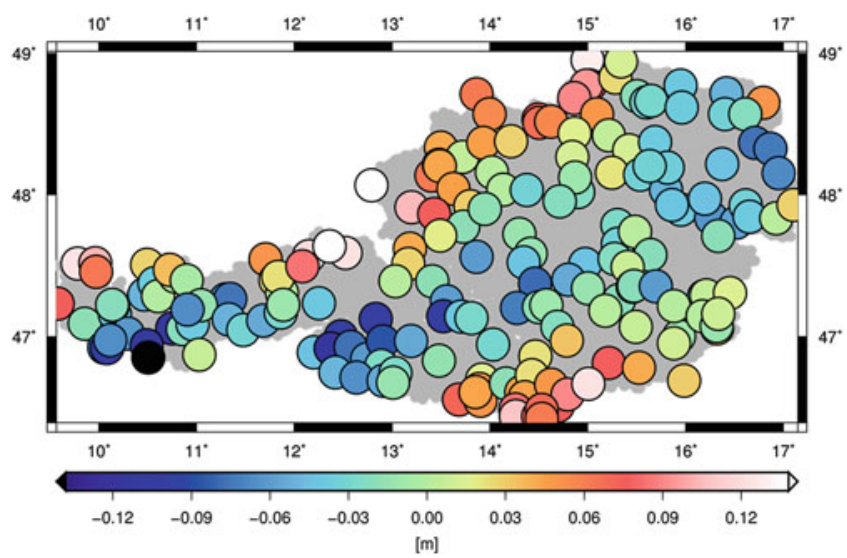

Fig. 9 Residuals between the computed gravimetric geoid and GPS/leveling observations; $r \mathrm{rms}=5.8 \mathrm{~cm}$

the estimated gravity based on RBF parametrization can be seen. Apart from a few outliers the quality of the input data is quite good. Additionally the point distance of the RBF is still insufficient and a densification is needed. In Table 2 the statistics of the residuals is shown. For this first investigation the outliers have not been eliminated and are still contained in the computed gravimetric geoid solution.

To check the quality of the pure gravimetric geoid solution GPS/leveling observation with measured geoid heights have been used (see Sect. 2.3). The resulting rms is lower than $6 \mathrm{~cm}$. It has to be remarked that the huge residuals are located near Austrian borders as shown in Fig. 9. This has to be expected due to edge effects and suggests that additional gravity data from neighbouring countries have to be taken into account to sustain the solution in these areas. 
Table 3 Statistics of 192 geoid height residuals-mean value was subtracted beforehand

\begin{tabular}{lllll}
\hline & Min $[\mathrm{cm}]$ & Max $[\mathrm{cm}]$ & Mean $[\mathrm{cm}]$ & $\mathrm{rms} \mathrm{[cm]}$ \\
\hline$v_{N}$ & -15.11 & 24.52 & 0.00 & 5.80 \\
\hline
\end{tabular}

The statistics of the geoidal height residuals can be seen in Table 3.

\section{Summary and Outlook}

The methodological improvements in Remove Compute Restore procedure result in a consistent combination of satellite and terrestrial data. Relative to previous Austrian geoid computation the long-wavelength errors of the gravimetric geoid are reduced compared to GPS/leveling observations and a correction surface is not needed anymore. An additional benefit results from the fact that the inhomogeneous distribution of the observations is not crucial compared to LSC and the full information provided by the GOCO model is incorporated. It has to be pointed out that the achieved results are at an early stage of computation and further improvement have to be done. This improvements are in particular:

- applying atmospheric corrections to measured gravity field quantities

- use of global DTM model (e.g. DTM 2006)

- use additional gravity data from neighbouring countries

The atmospheric correction of the measured gravity data will contribute to a slightly improvement of the reduced quantities. The use of a global DTM in addition to the currently available data will ensure a global DTM coverage and hence a global representation of DTM derived spherical harmonics. Additional data from neighbouring countries will sustain the solution and help to avoid edge effects. Furthermore a combined solution with deflections of the vertical $\xi, \eta$ is intended to achieve improvements. The optimum weighting between the observation groups will be performed by variance component estimation (Koch and Kusche 2002).

\section{References}

Drinkwater M, Floberghagen R, Haagmans R, Muzi D, Popescu A (2003) GOCE: ESAs first earth explorer core mission. Space sciences series of ISSI, vol 18. Kluwer Academic, Boston, pp 419-432. ISBN 1-4020-1408-2

Drinkwater M, Floberghagen R, Haagmans R, Muzi D, Kern M (2008) GOCE: Obtaining a portrait of earth's most intimate features. ESA Bull 133:4-13

Eicker A (2008) Gravity field refinement by radial basis functions from in-situ satellite data. Dissertation, Universitaet Bonn, Bonn
Graf J (1996) Das digitale Gelaendemodell fuer Geoidberechnungen und Schwerereduktionen in Oesterreich. In: Proceedings of the $7^{\text {th }}$ international meeting on alpine gravimetry-Heft 14, Wien, pp 121136. ISSN 1016-6254

Koch K, Kusche J (2002) Regularization of geopotential determination from satellite data by variance components. J Geodesy 76:259-268 (Bonn)

Kuehtreiber N, Pail R, Wasle B, Pock C, Wirnsberger H, HofmannWellenhof B, Steinbach O, Hoeggerl N, Imrek E, Ruess D, Ullrich C (2011) Improved Austrian geoid solution combining terrestrial and satellite gravity data (GEOID+). ASAP Phase VI Project-Final Report. https://online.tugraz.at, Graz

Mayer-Guerr T, Rieser D, Hoeck E, Brockmann J, Schuh WD, Krasbutter I, Kusche J, Maier A, Krauss S, Hausleitner W, Baur O, Jaeggi A, Meyer U, Prange L, Pail R, Fecher T, Gruber T (2012) The new combined satellite only model GOCO03s. In: Proceedings of the international symposium on gravity geoid and height systems GGHS 2012, Venice, Presentation-www.goco.eu

Moritz H (1980a) Advanced physical geodesy. Herbert Wichmann Verlag, Karlsruhe

Moritz H (1980b) Geodetic reference system 1980. Bull Geodes $55: 395-405$

Pail R, Kuehtreiber N, Wiesenhofer B, Hofmann-Wellenhof B, Of G, Steinbach O, Hoeggerl N, Ruess D, Ullrich C (2008) The new Austrian geoid solution (GEOnAUT). In: Gravity geoid and Earth observation 2008-International Association of Geodesy (IAG). https://online.tugraz.at

Pail R, Kuehtreiber N, Wiesenhofer B, Hofmann-Wellenhof B, Ullrich C, Hoeggerl N, Imrek E, Ruess D (2009) The official Austrian geoid solution 2008: data method and results. EGU General Assembly. https://online.tugraz.at, Vienna

Reigber C, Luehr H, Schwintzer P (2002) CHAMP mission status. Adv Space Res 30(2):129-134. doi:10.1016/S0273-1177(02)00276-4

Ruess D (1983) Ein digitales Höhenmodell von Österreich. Geodätische Arbeiten Österreichs für die internationale Erdmessung, Band III, Wien, pp 153-157

Rummel R, Rapp RH, Suenkel H, Tscherning CC (1988) Comparisons of global topographic/isostatic models to the Earth's observed gravity field. Department of Geodetic Science and Surveying Rep. No 388. The Ohio State University Columbus, Columbus

Schmidt H (1981) Sampling function and finite element method representation of the gravity field. Rev Geophys 19(3):421-436. doi:10.1029/RG019i003p00421

Tapley B, Bettadpur S, Watkins M, Reigber C (2002) The gravity recovery and climate experiment. Mission overview and early results. Geophys Res Lett 31:L09607. doi:10.1029/2004GL019920

Wild-Pfeiffer F (2007) Auswirkungen topographisch-isostatischer Massen auf die Satellitengradiometrie. Dissertation zu Karlsruhe Karlsruhe. ISBN 3769650423 